\section{Darmkrebs-Vorsorge: Stuhltests bleiben wichtige Alternative zur Endoskopie}

\begin{abstract}
Gesetzlich Krankenversicherte ab 55 Jahren haben die Wahl: entweder zweimal im Abstand von zehn Jahren eine Screening-Koloskopie oder alle zwei Jahre einen Stuhltest auf okkultes Blut. Wie wichtig diese Alternative ist, belegen die Ergebnisse einer Studie aus den Niederlanden.
\end{abstract}

\begin{abstract}
n der Studie wurden 8.407 Männer und
Frauen ab 50 Jahren schriftlich zu einer Screening-Sigmoidoskopie eingeladen und sechs Wochen später erinnert. Trotzdem wurde nur eine Beteiligungsquote von $31 \%$ erreicht. Daraufhin wurden 4.407 „Verweigerer“ erneut angeschrieben, mit dem Angebot eines immunologischen Stuhltests. $25 \%$ waren dazu bereit. Durch die zweistufige Einladung wurde die Beteiligungsrate auf $45 \%$ erhöht. Damit lag sie immer noch unter der Quote von $62 \%$, die in derselben Zielgruppe mit einer direkten Einladung zum Okkultblut-
\end{abstract}

Test erreicht worden war. Die diagnostische Ausbeute war, gemessen an der Zahl der Personen mit fortgeschrittenen Neoplasien, mit der Sigmoidoskopie und mit dem kombinierten Verfahren höher als mit der primären Stuhluntersuchung: Die Detektionsraten von fortgeschrittenen Adenomen lagen bei 7,4, 6,1 und 3,5\%, die von kolorektalen Karzinomen bei 0,6, 0,5 und 0,3\%. Mit der primären Sigmoidoskopie mussten 14 bzw. 172 Patienten untersucht werden, um ein fortgeschrittenes Adenom bzw. Karzinom zu entdecken. Bei dem zweistufigen Vorgehen waren dafür 16 bzw. 208 Screeningteilnehmer notwendig. Gründe für die Nichtakzeptanz von endoskopischen Untersuchungen sind einer italienischen Studie zufolge vor allem die Angst vor Schmerzen, das unangenehme Prozedere und die Furcht vor Verletzungen [Pignone $\mathrm{M}$ et al. J Gen Intern Med. 1999;14:432-7].

Fazit: In der Studie war jeder vierte Patient, der eine Einladung zur Sigmoidoskopie ausschlug, bereit, einen Stuhltest auf Okkultblut durchzuführen. Um den Erfolg von Darmkrebsvorsorge-Programmen zu verbessern, empfehlen die Autoren deswegen, einen Okkultblut-Test anzubieten, wenn die (effektivere) Koloskopie abgelehnt wird. Beate Schumacher

Hol L et al. Uptake of faecal immunochemical test screening among nonparticipants in a flexible sigmoidoscopy screening programme. Int J Cancer. 2012;130(9):2096-102.

\title{
Analkarzinom-Screening für homosexuelle Männer?
}

\begin{abstract}
Mehr als $80 \%$ aller Analkarzinome werden durch Infektionen mit humanen Papillomaviren (HPV) verursacht. Da homosexuelle Männer zu den Hochrisikogruppen gehören, gibt es den Vorschlag, für sie ein Karzinom-Screening anzubieten. Allerdings weiß man noch wenig über Häufigkeit und Verlauf der analen HPV-Infektion. Australische Wissenschaftler haben die bisher veröffentlichten Daten zusammengetragen.
\end{abstract}

nsgesamt werteten sie für die Metaanalyse 53 Studien aus, in denen homosexuelle Männer mit und ohne HIV-Infektion untersucht worden waren. Eine anale Infektion mit irgendeinem HPV-Typ fand sich bei 63,9\% der HIV-Infizierten und 92,6 \% der Männer ohne HIV-Infektion. Der riskante Virustyp 16 wurde bei 35,4 bzw. 12,5\% nachgewiesen. Hochgradige anale intraepitheliale Neoplasien (AIN) kamen bei den Männern mit HIV-Infektion ebenfalls häufiger vor (29,1 vs. $21,5 \%$ ohne HIV-Koinfektion). Analkarzinome wurden pro Jahr und 100.000 Männern 45,9- bzw. 5,1-mal diagnostiziert. Nach Einführung der antiretroviralen HIVTherapie lag die Krebsrate bei den HIVInfizierten etwas höher, möglicherweise aufgrund der längeren Lebenserwartung. Die Autoren kalkulierten daraus jährliche

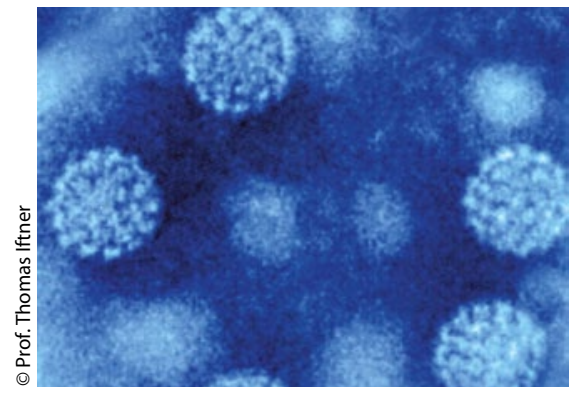

Das Risiko für eine HPV-Infektion im Analbereich ist bei Sex unter Männern erhöht.

Progressionsraten: Bei HIV-Positiven entwickelte sich ca. eine von 600 fortgeschrittenen Neoplasien zu einem Analkarzinom (in der HAART-Ära zirka eine von 400), bei HIV-negativen Patienten nur eine von ungefähr 4.000 Neubildungen. Die Progressionsrate ist damit niedriger als bei zervikalen intraepithelialen Neoplasien (CIN) im Stadium 3 (ungefähr eine von 80). Ob die Entfernung einer hochgradigen AIN vor Analkrebs schützt, ist noch gar nicht gezeigt. Damit, so die Autoren, ließen sich auch die Screening-Strategien für das Zervixkarzinom nicht einfach auf das Analkarzinom übertragen. Derzeit sollten Screeninguntersuchungen, genau wie die Behandlung hochgradiger AIN, der Forschung vorbehalten bleiben.

Fazit: Bei Männern, die Sex mit Männern haben, besteht fast immer eine HPV-Infektion im Analkanal; bei 20-30\% lassen sich hochgradige AIN nachweisen. Besonders hoch ist die Rate an auffälligen Befunden sowie Analkarzinomen bei HIV-infizierten homosexuellen Männern. Wegen der geringen Progressionsrate von fortgeschrittenen AIN zu Analkarzinomen ist der Nutzen eines Screenings fraglich.

Beate Schumacher

Machalek DA et al. Anal human papillomavirus infection and associated neoplastic lesions in men who have sex with men: a systematic review and meta-analysis. Lancet Oncol. 2012; 13(5):487-500. [Epub ahead of print]. - Kommentar: Wentzensen N. Screening for anal cancer: endpoints needed. Ibidem 438-40. 